\title{
Cell-nonautonomous inhibition of radiation-induced apoptosis by dynein light chain 1 in Caenorhabditis elegans
}

\author{
TH Morthorst ${ }^{1}$ and A Olsen ${ }^{*, 1}$
}

The evolutionarily conserved process of programmed cell death, apoptosis, is essential for development of multicellular organisms and is also a protective mechanism against cellular damage. We have identified dynein light chain 1 (DLC-1) as a new regulator of germ cell apoptosis in Caenorhabditis elegans. The DLC-1 protein is highly conserved across species and is a part of the dynein motor complex. There is, however, increasing evidence for dynein-independent functions of DLC-1, and our data describe a novel dynein-independent role. In mammalian cells, DLC-1 is important for cellular transport, cell division and regulation of protein activity, and it has been implicated in cancer. In C. elegans, we find that knockdown of dlc-1 by RNA interference (RNAi) induces excessive apoptosis in the germline but not in somatic cells during development. We show that DLC-1 mediates apoptosis through the genes lin-35, egl-1 and ced-13, which are all involved in the response to ionising radiation (IR)-induced apoptosis. In accordance with this, we show that IR cannot further induce apoptosis in dlc-1(RNAi) animals. Furthermore, we find that DLC-1 is functioning cell nonautonomously through the same pathway as kri-1 in response to IR-induced apoptosis and that DLC-1 regulates the levels of KRI-1. Our results strengthen the notion of a highly dynamic communication between somatic cells and germ cells in regulating the apoptotic process.

Cell Death and Disease (2013) 4, e799; doi:10.1038/cddis.2013.319; published online 12 September 2013

Subject Category: Cancer

Apoptosis is the process in which external or internal cues induce self-elimination of the cell. Apoptosis is an evolutionarily conserved key process in the development of multicellular organisms and is also implicated in the aetiology of several diseases such as cancer and neurodegeneration. ${ }^{1,2}$ In Caenorhabditis elegans, invariably, 131 somatic cells undergo apoptosis during development (developmental apoptosis) of the hermaphrodite, ${ }^{3,4}$ and in the adult half of the germ cells are eliminated by apoptosis (physiological germ cell apoptosis). ${ }^{5}$ These types of apoptosis in $C$. elegans depend on the core apoptotic machinery comprising the caspase CED- $3,{ }^{6}$ the adaptor protein Apaf-1 homologue CED $-4^{7}$ and the anti-apoptotic Bcl-2 homologue CED-9. ${ }^{8}$ Strong loss-of-function mutations in ced-3 or ced-4 as well as a gain-of-function (gf) mutation in ced- 9 completely inhibit apoptosis. ${ }^{5,9}$ DNA damage can also induce germ cell apoptosis (DNA damage-induced apoptosis) in the hermaphrodite germline. ${ }^{10}$ In addition to the core apoptotic machinery, DNA damage-induced apoptosis also depends on the tumour-suppressor p53 homologue CEP-1 $1^{11,12}$ and the BH3-only proteins EGL-1 and CED-13. ${ }^{13,14}$ All of these genes are functioning inside the dying cells to regulate the killing process. However, the genes vab-1 (ephrin receptor) and kri-1 (ankyrin-repeat protein orthologous to the human KRIT1/CCM1) have been shown to cell nonautonomously regulate physiological and ionising radiation (IR)-induced germ cell apoptosis, respectively. ${ }^{15,16}$

Apoptotic cells are removed by engulfment, and in the germline engulfment is carried out by the surrounding sheath cells. ${ }^{17}$ Two partially redundant pathways regulate engulfment. ${ }^{18}$ One pathway consists of the transmembrane receptor ced-1, the adaptor protein ced-6 (GULP) and ced-7 (ABC1). ${ }^{19-22}$ The other pathway comprises the adaptor protein ced-2 (Crkll), the guanine nucleotide-exchange factors ced-5 (DOCK180) and ced-12 (ELMO) and ced-10 (RAC1). ${ }^{23-26}$ Mutations in several engulfment genes impair the removal of dead cells, which consequently persist longer. ${ }^{18}$

Cytoplasmic dyneins are multisubunit motor protein complexes associated with microtubules. Large heavy chains comprise the bulk of the dynein complexes and confer motor activity, whereas the intermediate and light chains are accessory subunits that bind cargo. ${ }^{27,28}$ The mammalian dynein light chain DYNLL1 is highly conserved with $95 \%$ homology to the $C$. elegans homologue DLC-1. DYNLL1 is implicated in dynein-regulating processes such as vesicular

\footnotetext{
${ }^{1}$ Department of Molecular Biology and Genetics, Aarhus University, Gustav Wieds Vej 10C, Aarhus C DK-8000, Denmark

${ }^{*}$ Corresponding author: A Olsen, Department of Molecular Biology and Genetics, Aarhus University, Gustav Wieds Vej 10C, Aarhus C DK-8000, Denmark. Tel: +45 3069 8155; Fax: +45 8612 3178; E-mail: ano@mb.au.dk

Keywords: dynein light chain 1; apoptosis; DNA damage; C. elegans

Abbreviations: C. elegans, Caenorhabditis elegans; DLC-1, dynein light chain 1; IR, ionising radiation; RNAi, RNA interference; GFP, green-fluorescent protein; DIC, differential interference contrast; PCR, polymerase chain reaction; qRT-PCR, quantitative real-time polymerase chain reaction; gf, gain-of-function; MAPK, mitogenactivated protein kinase; Gy, grey; Unc, uncoordinated; ORF, open reading frame; NGM, nematode growth medium; DTT, dithiothreitol; PBS, phosphate-buffered saline; HRP, horseradish peroxidase; CDNA, complementary DNA; DMEM, Dulbecco's Modified Eagle Medium

Received 12.12.12; revised 18.6.13; accepted 30.7.13; Edited by M Piacentini
} 
and protein transport, cell division, mitotic spindle formation and nuclear migration. ${ }^{29,30}$ DYNLL1 binds to a variety of proteins besides dynein including the following: the pro-apoptotic protein BimL, ${ }^{31}$ p53-binding protein $1^{32,33}$ and the cell cycle regulators Cdk2 and Ciz1. ${ }^{34}$ There is increasing evidence that DYNLL1 can act independent of its association with microtubules. ${ }^{35,36}$ Several interaction partners of DYNLL1 regulate cell viability, ${ }^{31,33,34}$ and DYNLL1 is overexpressed in breast tumours. ${ }^{37}$ In accordance with a high evolutionary conservation, the $C$. elegans homologue DLC-1 affects germ cell proliferation, and inactivation of $d l c-1$ by RNA interference (RNAi) in a tumour-promoting background results in hyperproliferating and polyploid germ cells. ${ }^{38}$

We previously conducted a whole-genome RNAi screen with a view of identifying genes conferring resistance to the chemotherapeutic drug hydroxyurea (HU) (unpublished). The genes identified in the screen were analysed for germ cell apoptosis, and RNAi against dynein light chain 1 (dlc-1) caused a significant increase in the number of apoptotic germ cells. In this study, we describe a novel role of dlc-1 in regulating IR-induced germ cell apoptosis by a cellnonautonomous function via kri-1. We show that DLC-1 is functioning via egl-1 and ced-13 independently of cep-1.

\section{Results}

Lack of dlc-1 induces germ cells to undergo apoptosis. To investigate the effect of dlc-1 inactivation on germ cell apoptosis, we treated worms with RNAi against dlc-1 and quantified apoptosis using differential interference contrast (DIC) microscopy and the CED-1::GFP (green-fluorescent protein) reporter. During the engulfment process, the transmembrane receptor CED-1 expressed in sheath cells clusters around apoptotic cells. ${ }^{19}$ CED-1::GFP (Plim-7: :ced-1::gfp, bcls39) can therefore be used to quantify apoptotic germ cells undergoing engulfment. The dlc1(RNAi) animals had significantly more GFP-positive cells than the controls (Figures $1 \mathrm{a}$ and $\mathrm{b}$ ). A significant increase in the number of apoptotic cells following RNAi against dlc-1 was also seen using DIC microscopy (Figures 1a and b). The RNAi treatment reduced the expression of $d l c-1$ with $80-90 \%$ compared with controls (Supplementary Figure S1).

We reasoned that the increased number of apoptotic cells in the dlc-1(RNAi) animals could be because of excessive germ cells undergoing apoptosis or an engulfment defect preventing their removal. The CED-1::GFP marker can only be used if proper internalisation of the dead cells is taking place during the engulfment process; consequently, the CED-1::GFP marker does not detect apoptotic cells in the engulfmentdefective mutants ced-5 and ced- $6 .{ }^{39}$ We confirmed that persistent cell corpses observable with DIC in ced-5(tm1950) and ced-6(tm1826) mutants could not be seen with the CED-1::GFP marker (Figure 1c). Therefore, as the apoptotic cells in dlc-1(RNAi) animals could be observed equally well with DIC and CED-1::GFP, RNAi against dlc-1 did not result in an engulfment defect. Thus, dlc-1(RNAi) animals do not phenocopy engulfment-defective mutants.

To investigate whether $d l c-1$ is also involved in somatic apoptosis during development, we analysed L1 larvae for the presence of persistent cell corpses in the head with DIC microscopy. Wild-type N2 larvae have no visible cell corpses, whereas engulfment mutants, such as ced-5(tm1950) and ced-6(tm1826), have many persistent cell corpses ${ }^{18}$ (and Figure 1d). Inactivation of dlc-1 in wild-type N2 worms did not result in persistent cell corpses, indicating that $d / c-1$ does not affect engulfment during development. Increased apoptosis during development can be seen in engulfment-defective mutant backgrounds, as the extra dead cells are not as efficiently removed as in wild-type worms. However, RNAi against $d l c-1$ had no effect on the number of corpses observed in ced-5 or ced-6 mutants (Figure 1d). This suggests that RNAi against dlc-1 does not affect somatic cell death or engulfment but specifically increases germ cell apoptosis.

DLC-1 affects apoptosis independently of the dynein motor complex. In addition to dlc-1, the $C$. elegans genome encodes five other light chains $(d / c-2-6)$. We speculated whether the observed apoptotic phenotype was specific for the inactivation of $d l c-1$ or whether it was a general phenotype for all the dynein light chains. Following their inactivation, none of the other five light chains resulted in an increased number of apoptotic germ cells (Figure 1e). Furthermore, we also investigated the effect on apoptosis after knockdown of the main dynein heavy chain, dhc-1. As RNAi against $d h c-1$ causes developmental arrest, we treated N2 wild-type worms with RNAi against $d h c-1$ from the L4 stage and scored apoptosis $48 \mathrm{~h}$ later using DIC, but no effect on germ cell apoptosis was observed (Figure 1f). This indicates that the increase in apoptotic cells is specific for $d / c-1$ and is not a general phenotype from a disrupted dynein complex.

Lack of dlc-1 induces apoptosis through CED-3, CED-9 and LIN-35. Strong loss-of-function mutations in ced-3 or ced-4 completely inhibit both somatic and germ cell apoptosis. We inactivated $d l c-1$ in a mutant harbouring the strong loss-of-function allele ced-3(n717) and expressing the CED-1::GFP marker. The ced-3(n717) mutation completely suppressed apoptosis because of RNAi against dlc-1 (Figure 2a). ced-3-independent apoptosis has been reported following RNAi against the inhibitor of cell death-1 (icd-1) gene, which encodes the beta-subunit of the nascent polypeptide-associated complex (bNAC). ${ }^{40}$ Therefore, as an additional control we treated ced-3(n717) mutants with icd-1 RNAi. In agreement with the previous report, we observed that ced-3 could not completely suppress apoptosis induced by RNAi against icd-1 (Supplementary Figure S2). Thus, inactivation of dlc-1 increases the number of apoptotic germ cells exclusively through the ced-3 pathway. As almost all types of apoptosis in $C$. elegans require the core apoptotic machinery, we next decided to use an epistasis analysis approach to determine the subtype of apoptosis influenced by RNAi against dlc-1.

Physiological germ cell death is independent of the core apoptotic machinery gene ced- $9 .^{5}$ We tested whether dlc1(RNAi)-induced apoptosis was independent of a ced-9 gf mutation (n1950). However, dlc-1(RNAi)-induced apoptosis was completely abolished in the ced-9(gf) mutant, indicating that $c e d-9$ was required for $d l c-1$ (RNAi) to induce apoptosis (Figure 2b). Thus, dlc-1 is not involved in physiological germ cell death. 


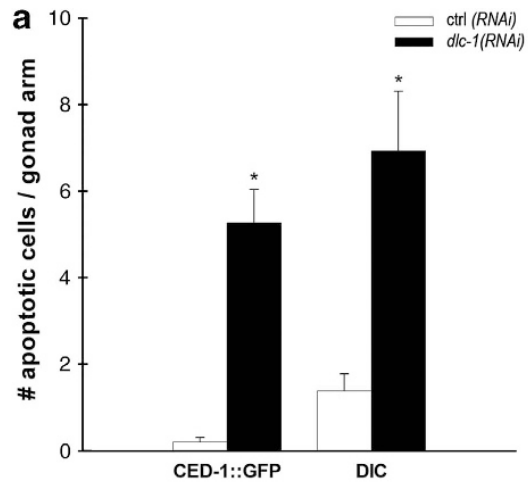

b
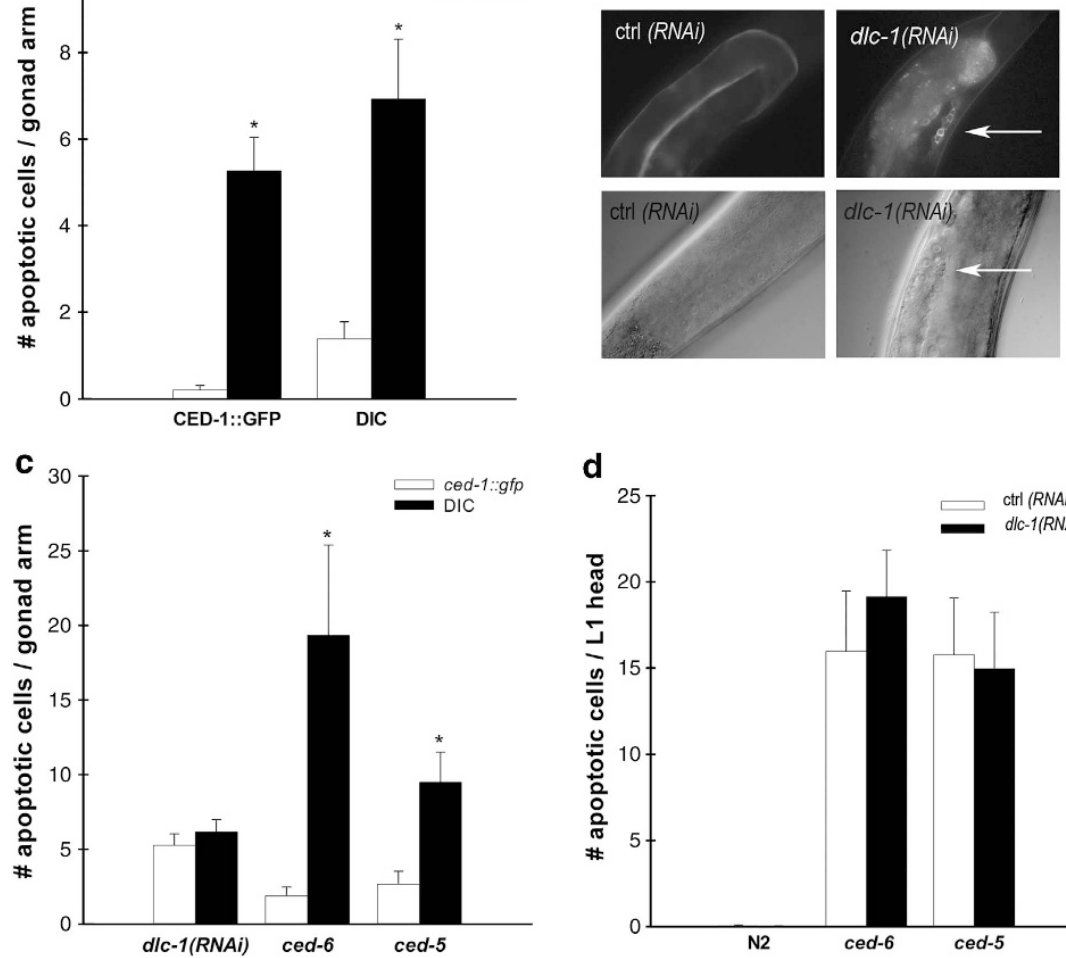

d
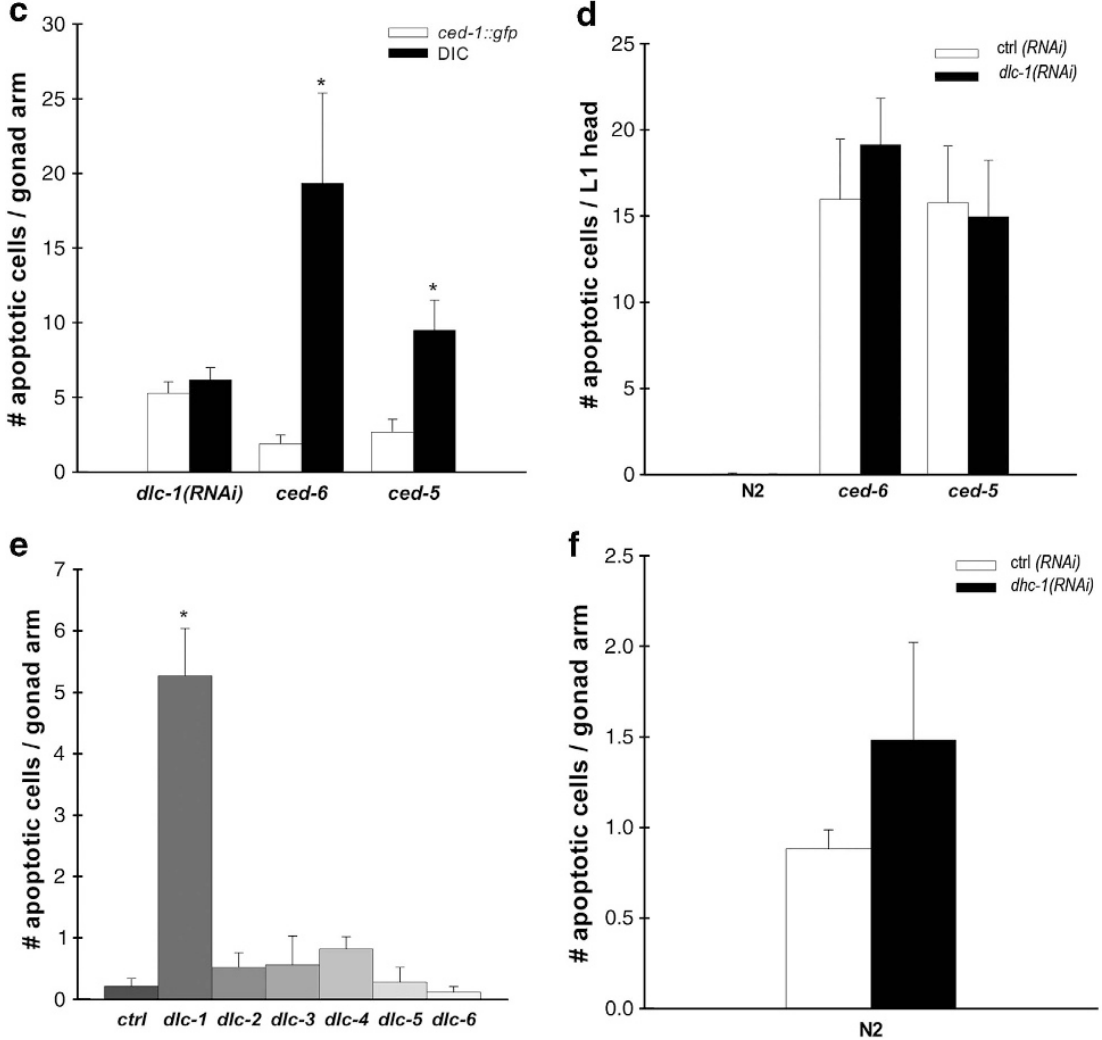

Figure 1 Inactivation of dlc-1 results in increased germ cell apoptosis independent of the dynein motor complex. (a) Mean number of apoptotic cells per gonad arm after RNAi against dlc-1, scored with both CED-1::GFP and DIC. (b) Epifluorescence $(\times 40)$ and DIC $(\times 40)$ pictures of ctrl and dlc-1 (RNAi) gonads. Arrows point to apoptotic cells. (c) Comparison between apoptosis scored with DIC and CED-1::GFP in dlc-1(RNAi), ced-6(tm1826) and ced-5(tm1950) animals. (d) Mean number of apoptotic cells in the head of freshly hatched L1 larvae in N2, ced-5(tm1950) and ced-6(tm1826) mutants treated with ctrl of dlc-1 RNAi, scored with DIC. (e) Mean number of apoptotic cells per gonad arm after RNAi against dlc-1-6, scored with CED-1::GFP. (f) Mean number of apoptotic cells per gonad arm in dhc-1(RNAi)-treated animals. All graphs are mean ( \pm S.D.) of at least three independent experiments. $N=20-40$ gonad arms for each experiment. ${ }^{*} P<0.05$

Inactivation of dlc- 1 results in a tumorous germline and unpaired chromosomes in the oocytes. ${ }^{38}$ Therefore, we hypothesised that these phenotypes might lead to DNA damage-induced apoptosis through CED-9. In C. elegans, DNA damage-induced apoptosis is dependent on the p53 homologue cep-1. ${ }^{11}$ Hence, we investigated whether dlc-1(RNAi) could induce apoptosis in cep-1(gk138) mutants. RNAi against dlc-1 induced apoptosis in the cep-1 mutants to the same extent as in wild-type N2 animals (Figure 2c). Thus, dlc-1(RNAi) does not result in apoptosis because of an increased level of DNA damage. Unpaired chromosomes can trigger a synapsis checkpoint independent of the DNA- damage checkpoint, which requires the activity of $\mathrm{PCH}-2$, the C. elegans homologue of $\mathrm{PCH} 2$, a budding yeast pachytene checkpoint gene. ${ }^{41}$ We found that inactivation of $d l c-1$ induced apoptosis in pch-2 mutants to a similar extent as seen in wild-type N2 animals (Figure 2d). Thus, dlc-1(RNAi) does not induce apoptosis because of unpaired chromosomes.

Several pathways have been shown to feed into the core apoptotic machinery through CED-9, such as the mitogen-activated protein kinase (MAPK) and retinoblastoma (RB) tumour-suppressor pathways. ${ }^{5,42}$ Overactivation of MAPK - for example, by loss of the inhibitor GLA-3 - causes excessive germ cell apoptosis. ${ }^{43}$ gla-3 mutants and dlc- 

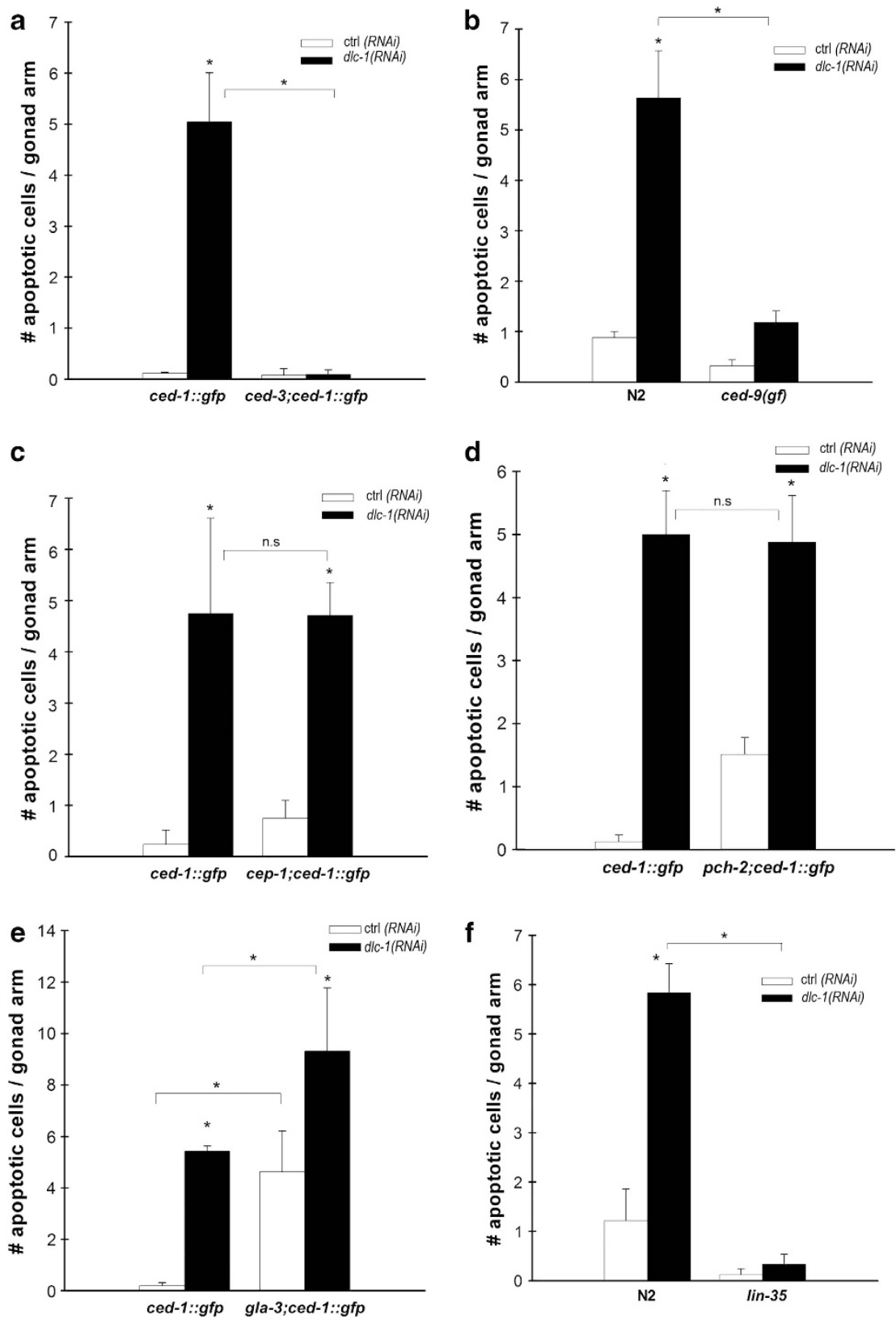

Figure 2 dlc-1(RNAi) induces apoptosis through CED-3, CED-9 and LIN-35 (a). Mean number of apoptotic cells per gonad arm after RNAi against dlc-1 in a ced-3(n717) mutant, scored with CE-1::GFP. (b) Mean number of apoptotic cells per gonad arm after RNAi against dlc-1 in a ced-9(n1950) mutant, scored with DIC. (c) Mean number of apoptotic cells per gonad arm after RNAi against dlc-1 in cep-1 (gk138) mutants, scored with CED-1::GFP. (d) Mean number of apoptotic cells per gonad arm after RNAi against $d l c-1$ in pch-2(tm1458) mutants, scored with CED-1::GFP. (e) Mean number of apoptotic cells per gonad arm after RNAi against dlc-1 in gla-3(ok2684) mutants, scored with CED-1::GFP. (f) Mean number of apoptotic cells per gonad arm after RNAi against dlc-1 in lin-35(n745) mutants, scored with DIC. All graphs are mean ( \pm S.D.) of at least three independent experiments. $N=20-40$ gonad arms for each experiment. ${ }^{*} P<0.05$

1(RNAi) animals had approximately equal levels of apoptosis, whereas the gla-3(ok2684); dlc-1(RNAi) animals had significantly higher levels of germ cell apoptosis than either of the single mutant/RNAi animals (Figure 2e), consistent with gla-3 and dlc-1 functioning in separate pathways to induce apoptosis. LIN-35, the $C$. elegans ortholog of the RB tumor-suppressor p105Rb, is involved in both physiological and DNA damage-induced germ cell apoptosis; however, contrary to gla-3, loss of lin-35 inhibits apoptosis. ${ }^{42}$ In lin-35(n745) mutants, RNAi against dlc-1-induced apoptosis was completely suppressed (Figure 2f). Thus, we conclude that normally DLC- 1 acts upstream of LIN-35 to protect against apoptosis. LIN-35 represses the transcription of ced- $9 ;^{42}$ hence, we hypothesised that DLC-1 could directly or indirectly affect the activity of LIN-35 and thereby the transcription of ced-9. We analysed the expression of lin-35 and ced-9 in dlc-1(RNAi) animals but observed no changes in their expression (Supplementary Figure S3), demonstrating 
that $d l c-1$ does not affect lin-35 and ced-9 activity at the transcriptional level.

DLC-1 is a part of the response to IR-induced apoptosis. As dlc-1(RNAi)-induced apoptosis was independent of cep-1 but dependent on lin-35, both of which are involved in response to DNA damage, we evaluated how dlc-1(RNAi) animals responded to DNA damage induced

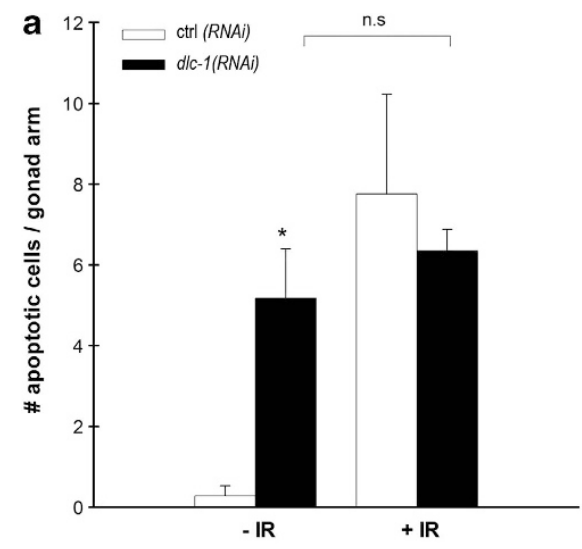

C
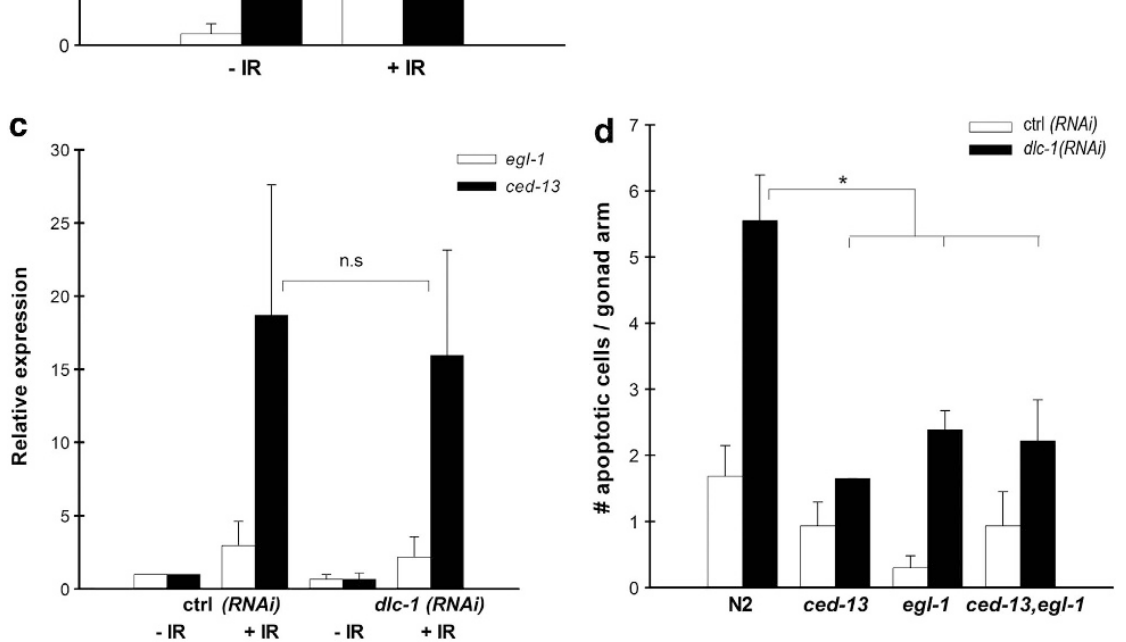

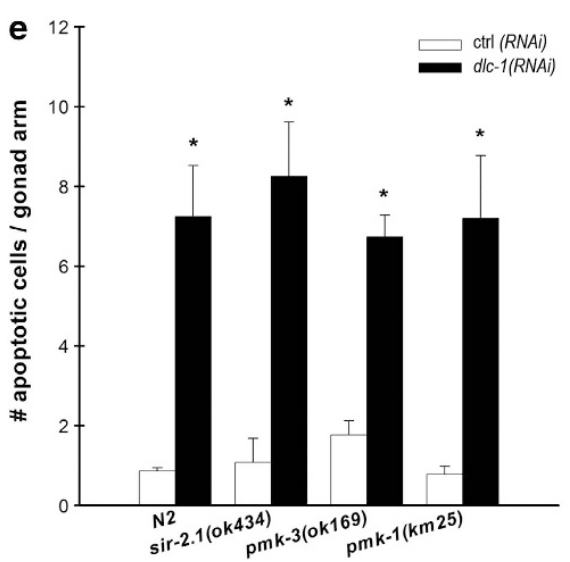

by IR. Interestingly, IR did not increase the number of apoptotic cells in dlc-1(RNAi) animals (Figure 3a), indicating that IR and dlc-1 function in a common pathway.

In human cells, DLC-1 facilitates the transport of p53 to the nucleus in response to DNA damage. ${ }^{32,33}$ The lack of response to IR in dlc-1(RNAi) animals could arise from the mislocalisation or malfunction of CEP-1. Using western

\section{b}

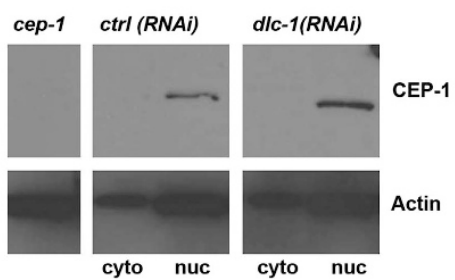


blotting with an antibody against the C-terminal of CEP-1, no difference in CEP-1 localisation was observed between control and dlc-1(RNAi) animals; in both CEP-1 was accumulated exclusively in the nucleus (Figure $3 \mathrm{~b}$ ). Furthermore, to evaluate the activity of CEP-1 in dlc-1(RNAi) animals, we used quantitative real-time polymerase chain reaction (qRT-PCR) to measure the expression levels of the CEP-1 target genes egl-1 and ced-13, which are upregulated after exposure to IR. ${ }^{14}$ In both the control and dlc-1(RNAi) animals, the expression levels of egl-1 and ced-13 were upregulated after exposure to IR, suggesting that CEP-1 functions correctly in dlc-1(RNAi) animals (Figure 3c). In contrast, cep-1 mutants could not induce egl-1 transcription in response to IR (Supplementary Figure S4).

We then investigated whether DLC-1 acted in the DNA-damage response pathway downstream or in parallel to cep-1. We quantified germ cell corpses using DIC in egl-1(n1084n3082);dlc-1(RNAi), ced-13(tm536);dlc-1(RNAi) and egl-1(n1084n3082);ced-13(tm536);dlc-1(RNAi) animals and found that dlc-1 RNAi-induced apoptosis was strongly reduced in these mutant backgrounds compared with RNAi against dlc-1 in a wild-type background (Figure $3 d$ ). Thus, both functional CED-13 and EGL-1 are required for RNAi against $d l c-1$ to induce apoptosis. However, RNAi against $d l c-1$ did not regulate the transcription of egl-1 and ced-13 in the absence of DNA damage (Figure 3c), suggesting that DLC-1 might regulate egl-1 and ced-13 at the protein level.

Other cep-1-independent apoptotic regulators have been described, including sir-2.1, ${ }^{44}$ pmk-1 $1^{45,46}$ and pmk-3. ${ }^{47}$ Therefore, we investigated the epistatic relationship between dlc-1 and these three genes. SIR-2.1 is a member of the Sirtuins family of $\mathrm{NAD}^{+}$-dependent protein deacetylases shown to regulated IR-induced apoptosis. The p38 homologues of mitogen-activated protein kinase, pmk-1 and pmk-3, are necessary for bacterial and copper-induced germ cell apoptosis, respectively. Neither sir-2.1, pmk-1 nor pmk-3 could suppress dlc-1(RNAi)-induced apoptosis, indicating that they act through different pathways (Figure $3 e$ ).

DLC-1 is functioning cell nonautonomously through kri-1. To study the expression and localisation of DLC-1, we generated two independent transgenic lines $\left(P_{\text {dlc }-1}:\right.$. dlc-1::gfp) expressing GFP-tagged DLC-1 under the regulation of the dlc-1 promotor (Figure 4). We found DLC-1 to be expressed in numerous tissues including those of the intestine (Figure 4a), body wall muscles (Figure 4b), germ cells and oocytes (Figure 4c), the rectal valve cell (Figure 4d) and unidentified cells in the head (Figure 4e). DLC-1 was expressed at all developmental stages from the one-cell embryo. To validate that the transgenic strain was expressing wild-type DLC-1, we treated it with RNAi against dlc-1 and observed that the GFP level was significantly reduced (Supplementary Figure S5), and we used western blotting to confirm that the full-length fusion protein was being expressed (data not shown).

As DLC-1 was expressed both in the germ cells and in somatic tissues, we investigated in which of these tissues inactivation of $d l c-1$ resulted in increased germ cell apoptosis. The $p p w$-1 gene encodes a member of the argonaute family of proteins and is required for efficient RNAi in germ cells. ${ }^{48}$
The $r r f-1$ gene encodes an RNA-directed RNA polymerase required for activation of the RNAi pathway in somatic cells. ${ }^{49}$ We treated two different alleles of rrf-1 (pk1417 and ok589) and ppw-1 (pk2505 and pk1425) with RNAi against dlc-1 and quantified the number of apoptotic cells using DIC. We observed that the increase in apoptotic cells caused by $d l c-1$ RNAi was completely abolished in the rrf-1 mutants but not affected in the ppw-1 mutants (Figure 5a). Therefore, inactivation of dlc-1 only in somatic cells gives rise to the increase in apoptotic cells. To verify the specificity of the rrf-1 and ppw-1 mutants, we treated them with ced-1 RNAi, which induces an engulfment defect. CED-1 functions in the somatic sheath cells but not in the germ cells. As expected, ced-1(RNAi) only induced an engulfment defect in ppw-1 mutants but not in rrf-1 mutants (Supplementary Figure S6).

As dlc-1 functions in the response to IR, we investigated whether IR regulates $d / c-1$ at the transcriptional or translational level. However, using qPCR no changes in dlc-1 transcription in response to IR was observed (data not shown). Likewise, we did not observe any changes in the levels of DLC-1::GFP after IR in whole animals (Figure 4f). We also examined the localisation of DLC-1::GFP after IR, but changes were observed neither in whole animals (Figure $4 \mathrm{~g}$ ) nor in sheath cells (Supplementary Figure S7). We also hypothesised that overexpression of DLC-1 might protect against IR-induced apoptosis. However, we observed no difference in the number of apoptotic cells between wild-type N2 and DLC-1::GFP animals when treated with 120 or 60 Gy (Figure 5b and Supplementary Figure S8).

The clear involvement of DLC-1 in somatic tissues in regulating germ cell apoptosis led us to investigate a possible involvement of kri-1. The $\mathrm{KRI}-1$ ankyrin-repeat protein is orthologous to the human KRIT1/CCM1, and it is required for IR-induced apoptosis in a novel pathway acting in somatic cells. ${ }^{16}$ We examined the epistatic relationship between $d l c-1$ and $k r i-1$ by treating kri-1(ok1251) mutants with RNAi against dlc-1. kri-1 mutants fed dlc-1 RNAi had significantly fewer apoptotic germ cells than wild-type N2 animals treated with RNAi against dlc-1 (Figure 5c). This indicates that kri-1 is necessary for dlc-1(RNAi)-induced apoptosis. Next, we used a strain expressing the functional KRI-1::GFP protein to examine whether dlc-1 could regulate the expression or localisation of KRI-1. We treated KRI-1::GFP animals with dlc-1 RNAi for 3 days and scored the expression of GFP compared with animals grown on control RNAi. Our results show that $d l c-1$ RNAi increases the expression of KRI-1::GFP on average with $57 \%$ (Figure $5 \mathrm{~d}$ ), demonstrating that dlc-1 can regulate $\mathrm{KRI}-1$. We did not observe any change in localisation of KRI-1::GFP because of RNAi against dlc-1 (Supplementary Figure S9). To test the possibility that DLC-1 and $\mathrm{KRI}-1$ might interact directly, we expressed $C$. elegans DLC-1 and KRI-1 in a mammalian two-hybrid system using luciferase as readout. As DLC-1 is known to bind to itself in mammalian cells, we used this interaction as a positive control (Figure 5e). However, we did not observe any interaction between DLC-1 and $\mathrm{KRI}-1$ (Figure $5 \mathrm{f}$ ). Based upon our results we propose that DLC-1 is regulating $\mathrm{KRI}-1$ in response to IRinduced apoptosis to mediate a pro-apoptotic signal from somatic cells to germ cells (Figure 6). 

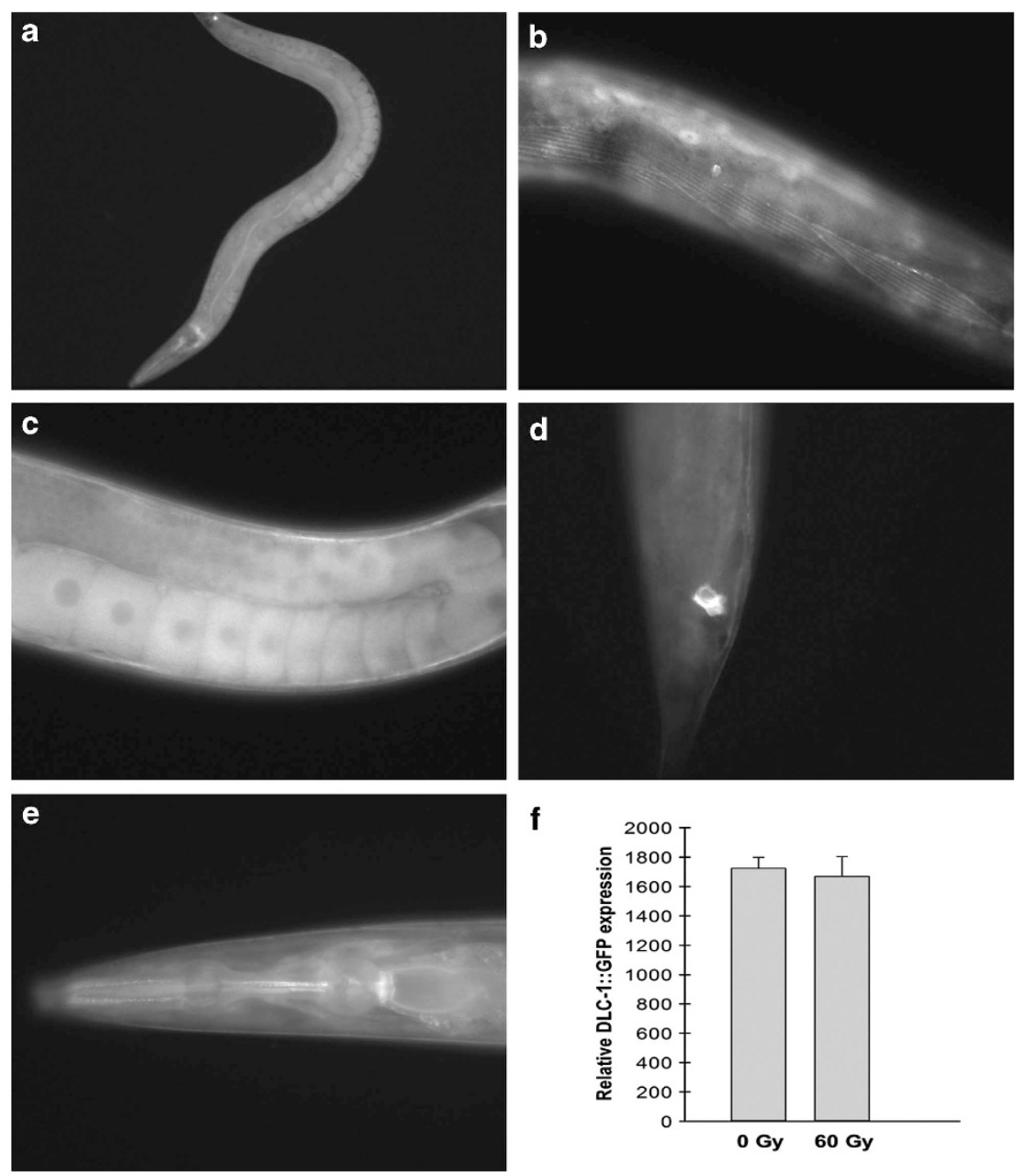

f
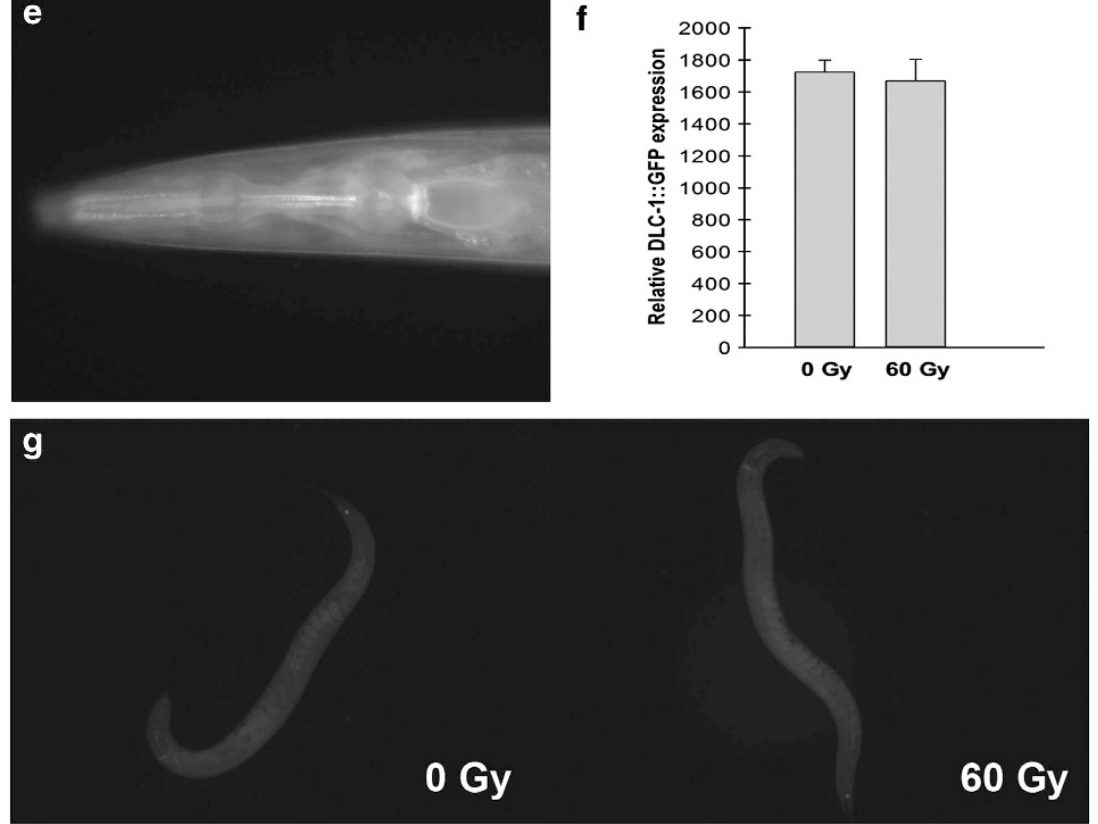

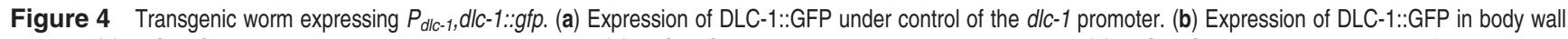
muscles. (c) DLC-1::GFP is expressed in germ cells and oocytes. (d) DLC-1::GFP is expressed in the rectal valve cells. (e) DLC-1::GFP expression in unidentified cells in the head. (f) Quantification (by ImageJ) of DLC-1::GFP expression after exposure to 0 and 60 Gy. (g) Expression of DLC-1::GFP $24 \mathrm{~h}$ after exposure to $60 \mathrm{~Gy}$

\section{Discussion}

We have identified DLC-1 as a new gene required for proper apoptotic signalling between somatic cells and germ cells in response to IR acting through $\mathrm{KRI}-1$. Our study is thus adding to the knowledge of the recently described KRI-1-mediated response to IR. Firstly, we find that KRI- 1 is required for CED3-dependent apoptosis induced by RNAi against dlc-1. Secondly, our data show that DLC-1 regulates the level of KRI-1. Thirdly, we show that the DLC-1/KRI-1 signalling pathway operates via EGL-1 and CED-13. Based upon these results we propose a model in which DLC-1 is part of the response to IR-induced apoptosis through a cell-nonautonomous pathway depending on kri-1 and independent of cep-1 (Figure 6). Consistent with this model we found that IR did not induce additional apoptosis in dlc-1(RNAi) animals. Our results strongly support that intercellular communication between somatic cells and germ cells is required for proper regulation of the cell-killing process. 

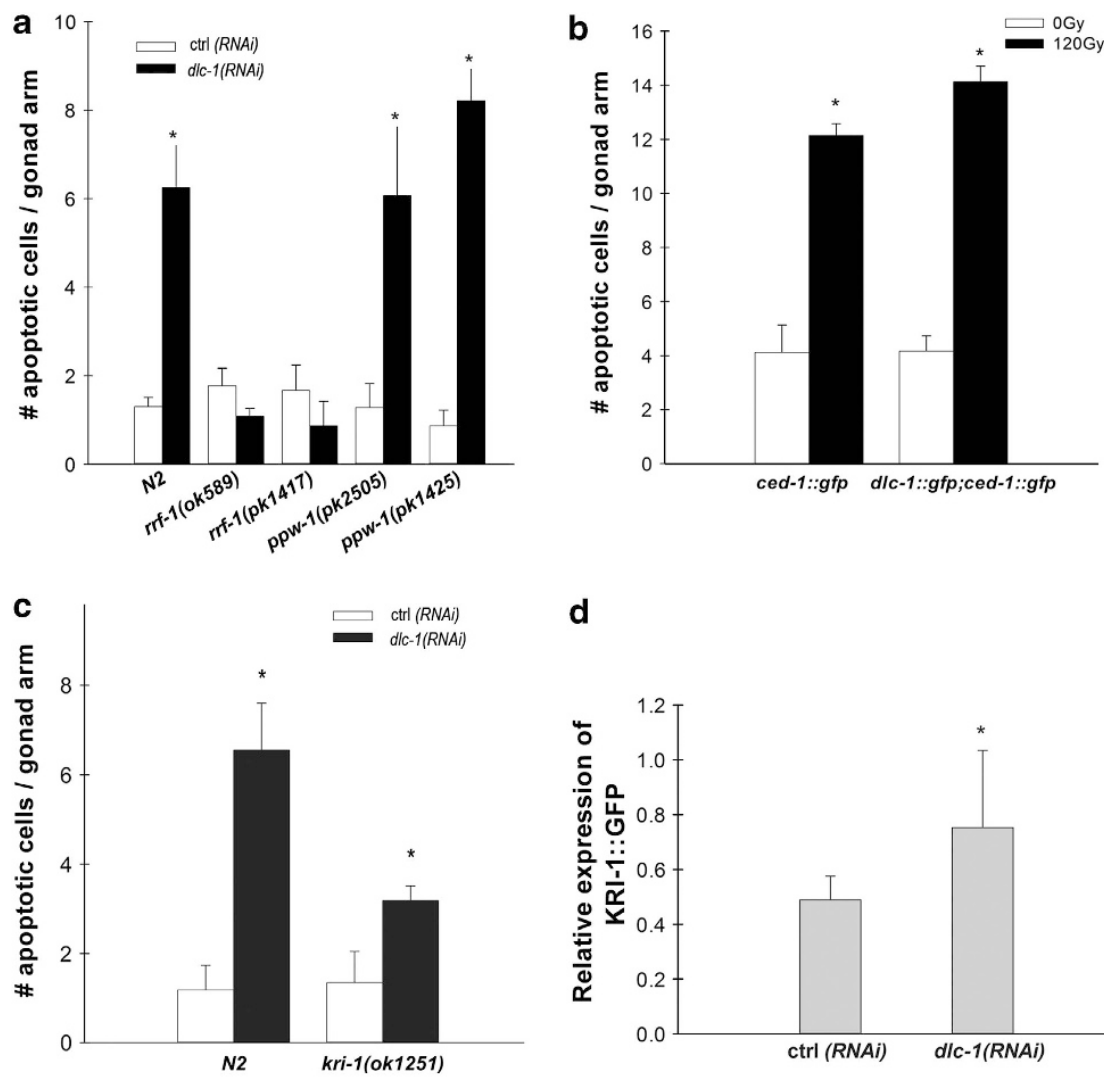

d
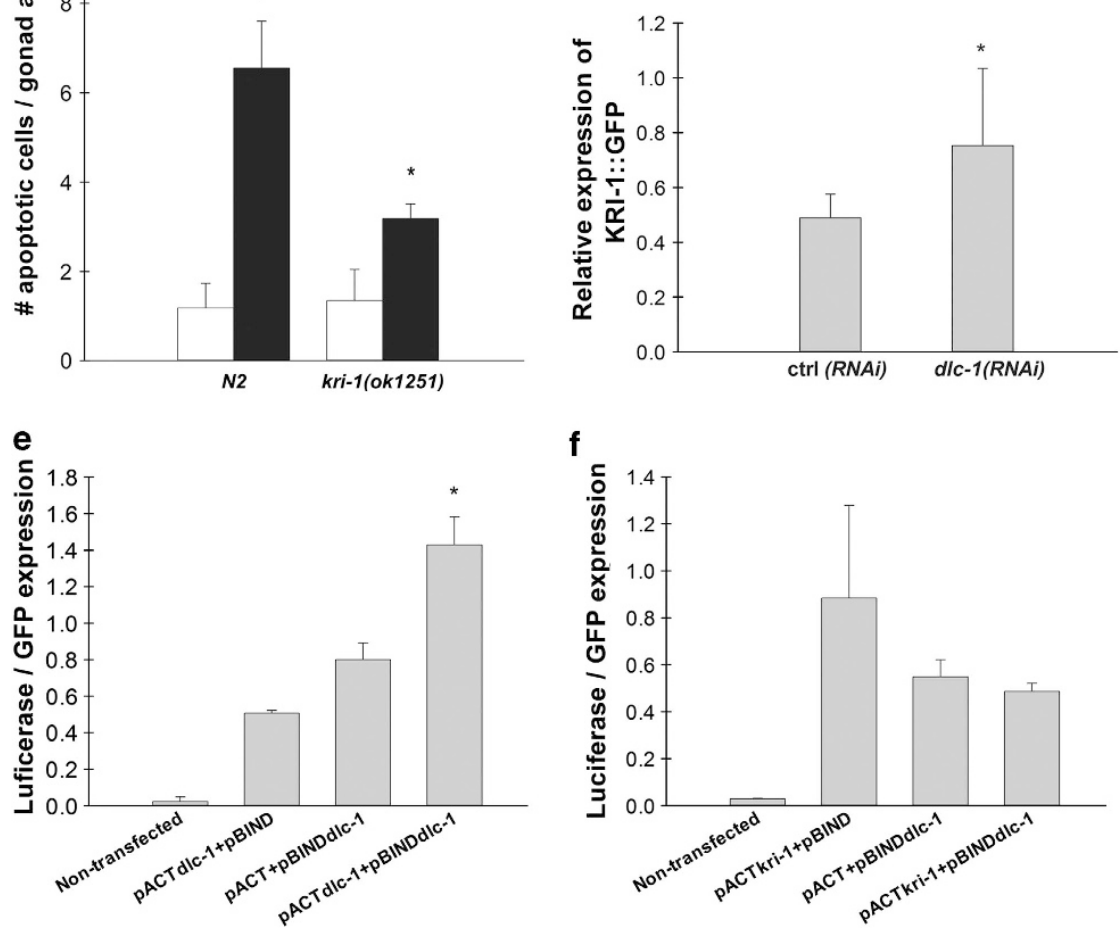

Figure 5 DLC-1 is functioning cell nonautonomously through kri-1. (a) Mean number of apoptotic cells per gonad arm after RNAi against dlc-1 in N2, ppw-1 (pk2505) and rrf-1(pk1417) mutants, scored with DIC. (b) Mean number of apoptotic cells per gonad arm in $P_{\text {dlc- }-1}$, dlc-1::gfp $24 \mathrm{~h}$ after treatment with 120 Gy, scored with CED-1::GFP. (c) Mean number of apoptotic cells per gonad arm after RNAi against dlc-1 in N2 and kri-1(ok1251) mutants. All graphs for apoptosis are mean ( \pm S.D.) of at least three independent experiments. For the apoptosis assays, $N=20-40$ gonad arms for each experiment. ${ }^{*} P<0.05$. (d) Expression of KRI-1::GFP after treatment with dlc-1 RNAi for 3 days. Quantified by ImageJ. Representative of three independent experiments. (e) DLC-1-DLC-1 interaction by the use of mammalian two-hybrid system. Representative of three independent experiments (f). DLC-1-KRI-1 interaction by use of mammalian two-hybrid system

Our model raised the possibility that dlc-1 was directly regulated by $\mathrm{IR}$ at the transcriptional or translational level. However, we did not observe any changes in expression or localisation of DLC-1::GFP in response to IR. Instead, IR may induce post-translational modifications to DLC-1. In mammalian cells, DLC-1 can be phosphorylated on Ser88 that inactivates DLC- 1 by disrupting the homodimer. ${ }^{50}$ This phosphorylation can be carried out by the p21-activated kinase, Pak-1. ${ }^{37}$

We have demonstrated that dlc-1 (RNAi)-induced apoptosis is dependent on EGL-1 and CED-13; however, DLC-1 is not involved in transcriptional regulation of egl-1 and ced-13. Rather our model predicts a second layer of regulation of the proteins involved in IR-induced apoptosis; CEP-1 regulates the transcription of egl-1 and ced-13, whereas DLC-1 indirectly regulates their activity at the protein level through a cell-nonautonomous pathway, giving a tighter and more fine-tuned regulation of IR-induced apoptosis.

At the molecular level, several mechanisms may explain how DLC-1 regulates apoptosis. One possible underlying molecular mechanism could be that DLC-1 normally binds a pro-apoptotic signal/protein. dlc-1 inactivation or exposure to 


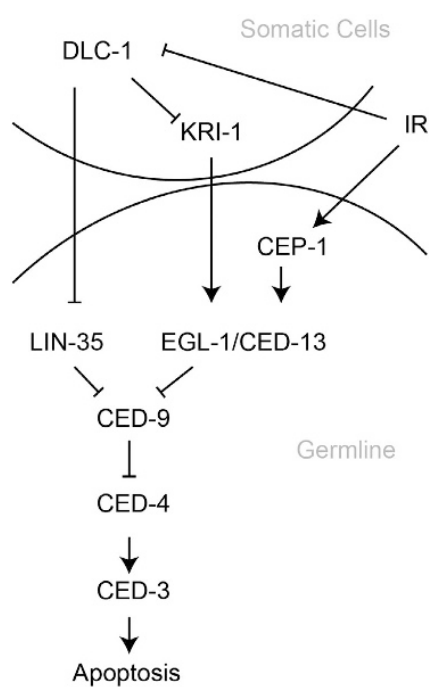

Figure 6 Model for the role of DLC-1 in regulation of germ cell apoptosis. Our data demonstrate that $\mathrm{DLC}-1$ acts cell nonautonomously through the kri-1 pathway in response to IR by indirectly regulating the activity of the proteins EGL-1, CED-13 and LIN-35 to induce germ cell apoptosis

IR then leads to the release of inhibition of such pro-apoptotic signal that is then transmitted to the germ cells to induce apoptosis by activating the EGL- 1 and CED-13 proteins. A similar cell-autonomous mechanism has been described in mammalian cells where DLC-1 binds to the BH3-only protein BimL and sequesters it to the dynein complex. Upon apoptotic stimuli, the DYNLL1-BimL dimer is released and translocated to the mitochondria to induce apoptosis. ${ }^{31}$ The cell-nonautonomous system that we are describing must contain an intermediate regulating protein, as DLC-1 and EGL-1/CED-13 are functioning in separate cells. This is not unlikely because mammalian $\mathrm{BH} 3-$ only proteins have been shown to be regulated post-translationally. ${ }^{51}$ Our data show that KRI-1 is one protein mediating the signalling from DLC-1 to EGI-1 and CED-13. We did not find experimental support for a direct interaction between DLC-1 and KRI-1; however, DLC-1 does regulate the level of $\mathrm{KRI}-1$. Further studies will be required to determine how and where DLC-1 is regulating KRI-1, and which downstream targets are employed in transmitting the apoptotic signal from the somatic cells to the germ cells. Interestingly, it has previously been shown that KRI-1 is expressed in the intestine and pharynx and that it mediates a signal between the reproductive system and the intestine required for longevity because of reduced germline signalling. ${ }^{52}$ As $d l c-1$ is also expressed in these tissues, it is tempting to speculate that they might be the site of interaction between DLC-1 and KRI-1, although their interaction also could be purely genetic and cell-nonautonomous.

Our results may also explain why DLC-1 is overexpressed in human breast cancers. ${ }^{37}$ It is possible that overexpression of DLC-1 could potentially protect cancer cells from undergoing apoptosis. Furthermore, our observation that DLC-1 mediates apoptotic signalling between somatic cells and germ cells validates the importance for cell-nonautonomous signalling. This intertissue communication also has vital roles in other $C$. elegans life history traits, such as lifespan ${ }^{52}$ and proliferation and differentiation of the germline..$^{53}$ Decoding pathways for intertissue communication is also of crucial importance in higher organism such as humans to better understand the interplay and regulation of individual organs required to maintain an intact organism.

\section{Materials and Methods}

Strains and culture conditions. All strains were maintained at $20^{\circ} \mathrm{C}$ on standard nematode growth medium (NGM) spotted with Escherichia coli strain OP50. The following strains were used: Wild-type N2, MD701 (bc/s39[P(lim-7) ced-1::GFP + lin-15(+)]), MT1522 ced-3(n717), ced-6(tm1826), MT4770 ced-9(n1950), FX536 ced-13(tm536), NL2098 rrf-1(pk1417), RB798 rrf-1(ok589), NL2550 ppw-1(pk2505), NL3511 ppw-1(pk1425), TJ1 cep-1(gk138), CA388 pch-2(tm1458), MT10430 lin-35(n745), RB2026 gla-3(ok2684), MT8735 egl-1(n1084n3082), ced-5(tm1950), egl-1(n1084n3082);ced-13(tm536), EG6699 (ttTi5605 II; unc-119 (ed3) III; oxEx1578), pmk-3(ok169), pmk-1(km25), sir-2.1(ok434), CF2052 kri-1(ok1251), muEx353[Pkri-1::kri-1::gfp; odr-1::Ifp], OLS400 aarSi1[Pdlc-1::DLC-1::GFP cb-unc-119(+)] II and OLS401 aarSi2[Pdlc$1:: D L C-1::$ GFP $c b-$ unc-119(+)] II.

Double mutants were made by crossing MD701 males with hermaphrodites of the desired phenotype. For cep-1;ced-1::GFP, pch-2;ced-1::GFP and gla-3;ced$1:: G F P$, the homozygous F2s were selected by using standard PCR (see Supplementary Table S1 for primers) and GFP expression. ced-3;ced-1::GFP was made by using the marker unc-26(e1196). The marker was first crossed to MD701, and F2s were selected by their Unc phenotype and GFP expression. This strain was then crossed to ced-3, and F2s were selected for GFP expression and non-Unc phenotypes. To validate the cep-1 mutant, we used IR to introduce DNA damage-induced apoptosis. As expected, the cep-1 mutant had no apoptotic cells in the germline after IR (Supplementary Figure S10).

Generation of transgenic strains by MosSCl. The plasmid containing

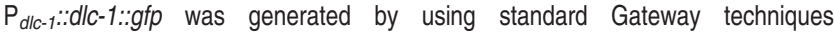
(Invitrogen, Naerum, Denmark). pENTRY clones for the dlc-1 promoter and open reading frame (ORF) were generated by using PCR products amplified with Gateway and gene-specific primers (Supplementary Table S1). The vector pJA256 (Addgene, Cambridge, MA, USA) was used as pENTRY clone for GFP. The Gateway destination vector was pCFJ150 (Addgene) containing an unc-119 rescue fragment.

MosSCl was performed as described ${ }^{54}$ using direct insertion based on injection of unc-119 animals (EG6699 strain) with an injection mix comprised of the plasmids

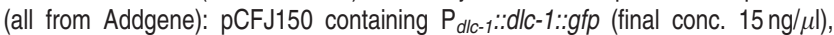
pCFJ601 (final conc. $50 \mathrm{ng} / \mu \mathrm{l}$ ), pMA122 (final conc. $10 \mathrm{ng} / \mu \mathrm{l}$ ), pGH8 (final conc. $10 \mathrm{ng} / \mu \mathrm{l}$ ), pCFJ90 (final conc. $2.5 \mathrm{ng} / \mu \mathrm{l}$ ) and pCFJ104 (final conc. $5 \mathrm{ng} / \mu \mathrm{l}$ ). Selection was based on peel-1 toxin, wild-type movement, absence of mCherry markers and presence of GFP.

Two independent lines OLS400 and OLS401 were created, and expression of full-length DLC-1 was confirmed by using western blotting with an antibody against GFP. The lines were subsequently back-crossed to wild-type N2 four times to eliminate background mutations.

RNAi. The RNAi clone against dlc-1(T26A5.9) was from the OpenBiosystems RNAi Library (Thermo Fisher Scientific, Waltham, MA, USA). RNAi clones against $d l c-2, d l c-3, d l c-4, d l c-5$ and $d l c-6$ were generated by standard cloning techniques and inserted into pL4440 and expressed by HT115 cells. All RNAi clones were verified by sequencing (See Supplementary Table S1 for primers). RNAi was performed by feeding on NGM plates containing $1 \mathrm{mM}$ isopropyl thiogalactoside and ampicillin $(100 \mu \mathrm{g} / \mathrm{ml}){ }^{55}$ Worms feed $\mathrm{HT} 115$ bacteria containing an empty pL4440 vector (ctrl RNAi) were used as controls.

In all germline apoptosis assays following RNAi against dlc-1, apoptosis was scored in the first generation on RNAi. For assays with RNAi against dlc-2-6, apoptosis was scored both in first and second generation on RNAi to eliminate maternal rescue. For dhc-1 RNAi, animals were treated with RNAi from L4 and apoptosis was scored $48 \mathrm{~h}$ later.

For developmental apoptosis in the head of $\mathrm{L} 1$ larvae on dlc-1 RNAi, eggs were picked onto dlc-1 RNAi plates and their progeny was scored for apoptosis.

Quantification of apoptosis. Cells undergoing apoptosis were distinguished from normal cells as refractile, button-like discs by using DIC microscopy 
(Zeiss Axiophot Microscope equipped with a Micropublisher 3.3 RTV Qimaging camera) as described. ${ }^{56}$ Alternatively, the CED-1::GFP ( $\mathrm{P}_{\text {lim- }-7 c e d-1:: G F P, ~ b c / s 39)}$ reporter was used to score apoptotic cells using fluorescence microscopy (Leica DNI300B microscope equipped with an Olympus DP72 camera) as previously described. ${ }^{19}$ Briefly, worms were synchronized as eggs and apoptosis was scored

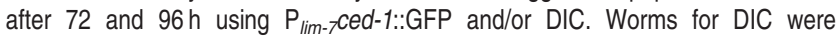
grown at $20^{\circ} \mathrm{C}$, whereas $\mathrm{P}_{\text {lim- }}$ - $C$ Ced-1::GFP worms were grown at $25^{\circ} \mathrm{C}$ to obtain stronger expression of GFP. Worms were anesthetised in $5 \mathrm{mM}$ levamisole in S-basal $\left(0.1 \mathrm{M} \mathrm{NaCl}, 0.05 \mathrm{M} \mathrm{H}_{2} \mathrm{PO}_{4}(\mathrm{pH} 6)\right)$ and mounted on $2 \%$ agarose pads. For DNA damage-induced apoptosis, hermaphrodites were synchronised as L4 and exposed to 60,90 or $120 \mathrm{~Gy}$ using a ${ }^{137} \mathrm{Cs}$ source (dose rate: $2 \mathrm{~Gy} / \mathrm{min}$ ). Germ cell apoptosis was scored 24-h post treatment. For quantification of developmental apoptosis, freshly hatched L1 larva was anaesthetised in $100 \mathrm{mM}$ levamisole in S-basal and mounted on $2 \%$ agarose pads.

Quantification of KRI-1::GFP expression Pictures of individual worms were taken with equal exposure time for ctrl and dlc-1(RNAi) worms. Pictures were converted to grey-scale and analysed by mean grey value in ImageJ (Rasband, W. S., Image J, US NIH, Bethesda, MD, USA). Twenty worms were analysed for each experiment and condition.

\section{Nuclear and cytoplasmic extracts and detection of CEP-1. Extracts} with minor modifications were prepared as described. ${ }^{57}$ Approximately 10000 rrf-3 worms were grown from eggs to adulthood on dlc-1 or empty vector RNAi plates. Worms were washed three times in S-basal. Three microlitre Extraction Buffer (10 mM Hepes, pH 7.1, $5 \mathrm{mM} \mathrm{MgCl}_{2}, 10 \%$ glycerol, $2 \mathrm{mM}$ dithiothreitol (DTT), Complete Protease inhibitor Cocktail Tablets (Roche Applied Science, Hvidover, Denmark)) was added before the worms were pelleted by centrifugation. The pellet was resuspended in 0.5 volumes of Extraction Buffer and snap-frozen in liquid nitrogen. The frozen worms were grinded in liquid nitrogen in a pre-chilled mortar and dissolved in $3 \mathrm{ml}$ Extraction Buffer and homogenised (10 strokes) in a Wheaton stainless steel tissue grinder (24-7). Samples were spun $10 \mathrm{~min}, 18000 \mathrm{~g}$ in $50 \mathrm{ml}$ tubes. The supematant (cytoplasmic fraction) was frozen in liquid nitrogen. The pellet was washed twice in $500 \mu \mathrm{l}$ Extraction buffer and then dissolved in $300 \mu \mathrm{l}$ Nuclear Extraction Buffer (Extraction Buffer with $500 \mathrm{mM} \mathrm{NaCl}$ ) and incubated for $30 \mathrm{~min}$, at $4^{\circ} \mathrm{C}$ on a roller table. After centrifugation for $10 \mathrm{~min}$, at $18000 \mathrm{~g}$ in $50 \mathrm{ml}$ tubes at $4^{\circ} \mathrm{C}$, the supernatant (nuclear fraction) was frozen in liquid nitrogen. Bio-Rad (Copenhagen, Denmark) sample buffer and reducing agent were added to $15 \mu \mathrm{l}$ of the samples, boiled for $5 \mathrm{~min}$ before being fractionated on Bio-Rad CriterionXT precast gels $(12 \%)$ and electroblotted onto a nitrocellulose membrane using a Bio-Rad Trans-blot cell and subsequently incubated with $2 \%$ skim milk in $1 \times$ phosphate-buffered saline (PBS). Membranes were probed with primary antibody followed by horseradish peroxidase (HRP)-conjugated secondary antibody. HRP was visualised using Amersham ECL plus detection systems (GE Healthcare, Brøndby, Denmark). Presence of CEP-1 in the extracts was detected with western blotting (standard protocol) using a rabbit anti-cep-1 (cN-18) antibody (Santa Cruz Biotechnology, Dallas, TX, USA) and HRP-conjugated swine anti-rabbit antibody (DakoCytomation, Glostrup, Denmark) as secondary antibody. An anti-actin antibody (JLA20, Calbiochem, Merck Chemicals, Hellerup, Denmark) was used as control for equal loading.

Ionising radiation. Hermaphrodites were synchronized at the L4 stage and irradiated with $90 \mathrm{~Gy}$ using a ${ }^{137} \mathrm{Cs}$ source (dose rate: $2 \mathrm{~Gy} / \mathrm{min}$ ). After radiation, the worms were transferred to fresh plates. Twenty-four hours post radiation, worms were washed in S-basal and then snap-frozen in liquid nitrogen and kept at $-80^{\circ} \mathrm{C}$.

Quantitative real-time PCR. For RNA isolation, Trizol (Invitrogen) and chloroform extraction were performed using Phase Lock Gel Heavy $2 \mathrm{ml}$ spin columns (5prime, VWR) followed by isopropanol precipitation. The quality of the RNA was assessed using gel electrophoresis and the concentration was measured using a NanoDrop 1000 (Thermo Scientific, Waltham, MA, USA). One microgram of total RNA was reverse-transcribed into complementary DNA (CDNA) using iScript cDNA Synthesis Kit (Bio-rad) following the manufacturer's protocol. Gene-specific qRT-PCR using CDNA, appropriate primers and Power SYBRGreen PCR Master Mix (Applied Biosystems, Naerum, Denmark), was performed on a Stratagene MX3000P machine (La Jolla, CA, USA). All samples were run in technical triplicates. Water was used as negative control. All primers were designed to span exon-exon boundaries (See Supplementary Table S1 for primers). Test gene $C_{\mathrm{T}}$ values were normalised to either act-1 (dlc-1, egl-1 and ced-13) or pmp-2 (lin-35 and ced-9) $C_{\mathrm{T}}$ values. Relative expression levels were calculated by using the Pfaffl method. ${ }^{58}$

Mammalian two-hybrid system. Experiments were performed based on the Checkmate Mammalian Two-Hybrid system (Promega, Madison, WI, USA). C. elegans KRI-1 (isoform a) and DLC-1 were cloned from whole-worm cDNA using standard cloning techniques (See Supplementary Table S1 for primers). KRI-1 was inserted into the PACT vector, and DLC-1 was inserted into both the pACT and pBIND vectors. HEK293T cells were seeded in 24-well plates with a density of $1.4 \times 10^{5}$ cells per well. Next day the cells were transfected at $70-90 \%$ confluence with $50 \mathrm{ng}$ GFP vector, $150 \mathrm{ng}$ pG5luc, $150 \mathrm{ng}$ pACT (with appropriate insert), $150 \mathrm{ng}$ pBIND (with appropriate insert) and $1 \mu$ l lipofectamine 2000 (Life Technologies, Naerum, Denmark) in $50 \mu \mathrm{l}$ DMEM serum-free medium (Life Technologies) per well. After $24 \mathrm{~h}$, the medium was refreshed. Fourty-eight hours after transfection, the cells were lysed, and $50 \mu$ l of lysate were transferred to 96well plates. GFP fluorescence was measured using a Perlkin Elmer Universal Microplate Analyzer Fusion-Alpha FPHT. Fourty microlitre luciferase (Promega) was added to each well, and fluorescence was measured in an Enspire 2300 Multi label Reader. Background levels from empty cells were subtracted and luciferase/ GFP was plotted as binding efficiency. All measurements were run in technical triplicates. Non-transfected cells, cells transfected with either PACT or pBIND without insert or both were used as negative controls.

\section{Conflict of Interest}

The authors declare no conflict of interest.

Acknowledgements. We thank the members of the Olsen lab, Dr. MK Lund, Dr. MK Larsen and Dr. M Hansen for their helpful discussions and feedback on the manuscript. For help with the mammalian two-hybrid system, we thank Dr. D Bhaumik. ced-5(tm1950) was obtained from Professor Mitani (Tokyo, Japan). egl-1(n1084n3082);ced-13(tm536) was kindly provided by Professor Shaham (The Rockefeller University, New York, NY, USA). muEx353[Pkri-1,kri-1::gfp; odr-1::Ifp] was kindly provided by Professor Kenyon (UCSF, CA, USA). All other nematode strains used in this work were provided by the Caenorhabditis Genetics Center, which is funded by the NIH National Center for Research Resources (NCRR). THM was supported by GSST, AO was supported by the Danish Research Council FNU (272-07-0162), The Danish Agency for Science, Technology and Innovation (2136-08-0007), The Interdisciplinary Research Consortium on Geroscience (UL1 RR024917), The Novo Nordic Foundation and The Lundbeck Foundation (R9-A968).

1. Fadeel B, Orrenius S. Apoptosis: a basic biological phenomenon with wide-ranging implications in human disease. J Intern Med 2005; 258: 479-517.

2. Hipfner DR, Cohen SM. Connecting proliferation and apoptosis in development and disease. Nat Rev Mol Cell Biol 2004; 5: 805-815

3. Sulston JE, Schierenberg E, White JG, Thomson JN. The embryonic cell lineage of the nematode Caenorhabditis elegans. Dev Biol 1983; 100: 64-119.

4. Sulston JE, Horvitz HR. Post-embryonic cell lineages of the nematode, Caenorhabditis elegans. Dev Biol 1977; 56: 110-156.

5. Gumienny TL, Lambie E, Hartwieg E, Horvitz HR, Hengartner MO. Genetic control of programmed cell death in the Caenorhabditis elegans hermaphrodite germline. Development 1999; 126: 1011-1022.

6. Yuan J, Shaham S, Ledoux S, Ellis HM, Horvitz HR. The C. elegans cell death gene ced-3 encodes a protein similar to mammalian interleukin-1 beta-converting enzyme. Cell 1993; 75: 641-652.

7. Yuan J, Horvitz HR. The Caenorhabditis elegans cell death gene ced-4 encodes a novel protein and is expressed during the period of extensive programmed cell death. Development 1992; 116: 309-320.

8. Hengartner MO, Horvitz HR. C. elegans cell survival gene ced-9 encodes a functional homolog of the mammalian proto-oncogene bcl-2. Cell 1994; 76: 665-676.

9. Ellis HM, Horvitz HR. Genetic control of programmed cell death in the nematode C. elegans. Cell 1986; 44: 817-829.

10. Gartner A, Boag PR, Blackwell TK. Germline Survival and Apoptosis (September 4, 2008), WormBook (ed.). The C. elegans Research Community, WormBook, doi:10.1895/ wormbook.1.145.1, http://www.wormbook.org.

11. Derry WB, Putzke AP, Rothman JH. Caenorhabditis elegans p53: role in apoptosis meiosis, and stress resistance. Science 2001; 294: 591-595. 
12. Schumacher B, Hofmann K, Boulton S, Gartner A. The C. elegans homolog of the p53 tumor suppressor is required for DNA damage-induced apoptosis. Curr Biol 2001; 11: $1722-1727$.

13. Gartner A, Milstein S, Ahmed S, Hodgkin J, Hengartner MO. A conserved checkpoint pathway mediates DNA damage-induced apoptosis and cell cycle arrest in C. elegans. Mol Cell 2000; 5: 435-443.

14. Schumacher B, Schertel C, Wittenburg N, Tuck S, Mitani S, Gartner A et al. C. elegans ced-13 can promote apoptosis and is induced in response to DNA damage. Cell Death Differ 2005: 12: 153-161

15. Li X, Johnson RW, Park D, Chin-Sang I, Chamberlin HM. Somatic gonad sheath cells and Eph receptor signaling promote germ-cell death in C. elegans. Cell Death Differ 2012; 19 : 1080-1089.

16. Ito $\mathrm{S}$, Greiss $\mathrm{S}$, Gartner $\mathrm{A}$, Derry WB. Cell-nonautonomous regulation of $C$. elegans germ cell death by kri-1. Curr Biol 2010; 20: 333-338.

17. Gumienny TL, Hengartner MO. How the worm removes corpses: the nematode $C$. elegans as a model system to study engulfment. Cell Death Differ 2001; 8: 564-568.

18. Ellis RE, Jacobson DM, Horvitz HR. Genes required for the engulfment of cell corpses during programmed cell death in Caenorhabditis elegans. Genetics 1991; 129: 79-94.

19. Zhou Z, Hartwieg E, Horvitz HR. CED-1 is a transmembrane receptor that mediates cell corpse engulfment in C. elegans. Cell 2001; 104: 43-56.

20. Liu QA, Hengartner MO. Candidate adaptor protein CED-6 promotes the engulfment of apoptotic cells in C. elegans. Cell 1998; 93: 961-972.

21. Su HP, Nakada-Tsukui K, Tosello-Trampont AC, Li Y, Bu G, Henson PM et al. Interaction of CED-6/GULP, an adapter protein involved in engulfment of apoptotic cells with CED-1 and CD91/low density lipoprotein receptor-related protein (LRP). J Biol Chem 2002; 277: 11772-11779.

22. Wu YC, Horvitz HR. The $C$. elegans cell corpse engulfment gene ced-7 encodes a protein similar to ABC transporters. Cell 1998; 93: 951-960.

23. Reddien PW, Horvitz HR. CED-2/Crkll and CED-10/Rac control phagocytosis and cell migration in Caenorhabditis elegans. Nat Cell Biol 2000; 2: 131-136.

24. Wu YC, Tsai MC, Cheng LC, Chou CJ, Weng NY. C. elegans CED-12 acts in the conserved crkll/DOCK180/Rac pathway to control cell migration and cell corpse engulfment. Dev Cell 2001; 1: 491-502.

25. Zhou Z, Caron E, Hartwieg E, Hall A, Horvitz HR. The C. elegans PH domain protein CED-12 regulates cytoskeletal reorganization via a Rho/Rac GTPase signaling pathway. Dev Cell 2001; 1: 477-489.

26. Wu YC, Horvitz HR. C. elegans phagocytosis and cell-migration protein CED- 5 is similar to human DOCK180. Nature 1998; 392: 501-504.

27. Vallee RB, Sheetz MP. Targeting of motor proteins. Science 1996; 271: 1539-1544.

28. Harrison A, King SM. The molecular anatomy of dynein. Essays Biochem 2000; 35: 75-87.

29. Vaisberg EA, Koonce MP, McIntosh JR. Cytoplasmic dynein plays a role in mammalian mitotic spindle formation. J Cell Biol 1993; 123: 849-858.

30. Holzbaur EL, Vallee RB. DYNEINS: molecular structure and cellular function. Annu Rev Cell Biol 1994; 10: 339-372.

31. Puthalakath H, Huang DC, O'Reilly LA, King SM, Strasser A. The proapoptotic activity of the $\mathrm{Bcl}-2$ family member Bim is regulated by interaction with the dynein motor complex. Mol Cell 1999; 3: 287-296.

32. Giannakakou P, Sackett DL, Ward Y, Webster KR, Blagosklonny MV, Fojo T. p53 is associated with cellular microtubules and is transported to the nucleus by dynein. Nat Cell Biol 2000; 2: 709-717.

33. Lo KW, Kan HM, Chan LN, Xu WG, Wang KP, Wu Z et al. The 8-kDa dynein light chain binds to p53-binding protein 1 and mediates DNA damage-induced p53 nuclea accumulation. J Biol Chem 2005; 280: 8172-8179.

34. den Hollander $\mathrm{P}$, Kumar R. Dynein light chain 1 contributes to cell cycle progression by increasing cyclin-dependent kinase 2 activity in estrogen-stimulated cells. Cancer Res 2006; 66: 5941-5949.

35. Rapali P, Radnai L, Süveges D, Harmat V, Tölgyesi F, Wahlgren WY et al. Directed evolution reveals the binding motif preference of the LC8/DYNLL hub protein and predicts large numbers of novel binders in the human proteome. PLoS One 2011; 6: e18818.

36. Barbar E. Dynein light chain LC8 is a dimerization hub essential in diverse protein networks. Biochemistry 2008; 47: 503-508.

37. Vadlamudi RK, Bagheri-Yarmand R, Yang Z, Balasenthil S, Nguyen D, Sahin AA et al. Dynein light chain 1, a p21-activated kinase 1-interacting substrate, promotes cancerous phenotypes. Cancer Cell 2004; 5: 575-585.
38. Dorsett M, Schedl T. A role for dynein in the inhibition of germ cell proliferative fate. Mol Cell Biol 2009; 29: 6128-6139.

39. Almendinger J, Doukoumetzidis K, Kinchen JM, Kaech A, Ravichandran KS, Hengartner MO. A conserved role for SNX9-family members in the regulation of phagosome maturation during engulfment of apoptotic cells. PLoS One 2011; 6: e18325.

40. Bloss TA, Witze ES, Rothman JH. Suppression of CED-3-independent apoptosis by mitochondrial betaNAC in Caenorhabditis elegans. Nature 2003; 424: 1066-1071.

41. Bhalla N, Dernburg AF. A conserved checkpoint monitors meiotic chromosome synapsis in Caenorhabditis elegans. Science 2005; 310: 1683-1686.

42. Schertel C, Conradt B. C. elegans orthologs of components of the RB tumor suppressor complex have distinct pro-apoptotic functions. Development 2007; 134: 3691-3701.

43. Kritikou EA, Milstein S, Vidalain PO, Lettre G, Bogan E, Doukoumetzidis K et al. C. elegans GLA-3 is a novel component of the MAP kinase MPK-1 signaling pathway required for germ cell survival. Genes Dev 2006; 20: 2279-2292.

44. Greiss S, Hall J, Ahmed S, Gartner A. C. elegans SIR-2.1 translocation is linked to a proapoptotic pathway parallel to cep-1/p53 during DNA damage-induced apoptosis. Genes Dev 2008; 22: 2831-2842.

45. Aballay A, Drenkard E, Hilbun LR, Ausubel FM. Caenorhabditis elegans innate immune response triggered by Salmonella enterica requires intact LPS and is mediated by a MAPK signaling pathway. Curr Biol 2003; 13: 47-52.

46. Pei B, Wang S, Guo X, Wang J, Yang G, Hang $\mathrm{H}$ et al. Arsenite-induced germline apoptosis through a MAPK-dependent, p53-independent pathway in Caenorhabditis elegans. Chem Res Toxicol 2008; 21: 1530-1535

47. Wang S, Wu L, Wang Y, Luo X, Lu Y. Copper-induced germline apoptosis in Caenorhabditis elegans: the independent roles of DNA damage response signaling and the dependent roles of MAPK cascades. Chem Biol Interact 2009; 180: 151-157.

48. Tijsterman M, Okihara KL, Thijssen K, Plasterk RH. PPW-1, a PAZ/PIWI protein required for efficient germline RNAi, is defective in a natural isolate of $C$. elegans. Curr Biol 2002; 12: $1535-1540$.

49. Sijen T, Fleenor J, Simmer F, Thijssen KL, Parrish S, Timmons $\mathrm{L}$ et al. On the role of RNA amplification in dsRNA-triggered gene silencing. Cell 2001; 107: 465-476.

50. Song C, Wen W, Rayala SK, Chen M, Ma J, Zhang M et al. Serine 88 phosphorylation of the $8-k D a$ dynein light chain 1 is a molecular switch for its dimerization status and functions. J Biol Chem 2008; 283: 4004-4013.

51. Puthalakath $\mathrm{H}$, Strasser A. Keeping killers on a tight leash: transcriptional and posttranslational control of the pro-apoptotic activity of BH3-only proteins. Cell Death Differ 2002; 9: 505-512.

52. Berman JR, Kenyon C. Germ-cell loss extends $C$. elegans life span through regulation of DAF-16 by kri-1 and lipophilic-hormone signaling. Cell 2006; 124: 1055-1068

53. Killian DJ, Hubbard EJ. C. elegans pro-1 activity is required for soma/germline interactions that influence proliferation and differentiation in the germ line. Development 2004; 131 : $1267-1278$.

54. Frokjaer-Jensen C, Davis MW, Hopkins CE, Newman BJ, Thummel JM, Olesen SP et al. Single-copy insertion of transgenes in Caenorhabditis elegans. Nat Genet 2008; 40: 1375-1383.

55. Timmons L, Fire A. Specific interference by ingested dsRNA. Nature 1998; 395: 854

56. Hedgecock EM, Sulston JE, Thomson JN. Mutations affecting programmed cell deaths in the nematode Caenorhabditis elegans. Science 1983; 220: 1277-1279.

57. Tops BB, Tabara H, Sijen T, Simmer F, Mello CC, Plasterk RH et al. RDE-2 interacts with MUT-7 to mediate RNA interference in Caenorhabditis elegans. Nucleic Acids Res 2005; 33: $347-355$

58. Pfaffl MW. A new mathematical model for relative quantification in real-time RT-PCR. Nucleic Acids Res 2001; 29: e45.

(i) $\Theta($ Cell Death and Disease is an open-access journal cy ${ }_{\mathrm{B}} \mathrm{ND}$ published by Nature Publishing Group. This work is licensed under a Creative Commons Attribution-NonCommercialNoDerivs 3.0 Unported License. To view a copy of this license, visit http://creativecommons.org/licenses/by-nc-nd/3.0/ 